\title{
Etude épidémiologique de la rhinopneumonie chez les équidés au Maroc
}

\author{
A. Hmidouch ${ }^{1 *}$ M. El Harrak ${ }^{2}$ A. Chakri ${ }^{1}$ \\ L. Ouragh ${ }^{1}$ C. Lotfi ${ }^{2}$ L. Bakkali-Kassimi ${ }^{2}$
}

\section{Mots-clés}

Cheval - Ane - Mulet - Equidae - Virus rhinopneumonie équine - Technique immunologique - Prévalence de la maladie - Surveillance épidémiologique Maroc.

\begin{abstract}
Résumé
Lors d'une enquête sérologique sur la rhinopneumonie équine au $\mathrm{M}$ aroc, 4775 prélèvements $(59,6$ p. 100 de chevaux, 18 p. 100 de mulets et 22,4 p. 100 d'ânes) ont été réalisés durant cinq ans (de 1990 à 1994) dans les différentes régions du pays. La séroprévalence globale de l'infection était de 39 p. 100. Les ânes (46 p. 100) étaient plus affectés que les mulets (42 p. 100) et les chevaux (35 p. 100). En outre, chez les trois espèces, la séroprévalence a augmenté avec l'âge jusqu'à trois ans pour atteindre la valeur de 42 p. 100 et elle s'est stabilisée pour les animaux plus âgés. II n'est pas certain que le sexe et la région géographique où vivaient les animaux les prédisposaient à la maladie. Par contre, leurs conditions d'entretien ont constitué un facteur prédisposant à l'infection. Après une stabilisation pendant les quatre premières années, l'incidence de l'infection s'est subitement accrue en 1994 à cause de la sécheresse qui a sévi cette année-là.
\end{abstract}

\section{INTRODUCTION}

La rhinopneumonie équine est une maladie infectieuse spécifique aux équidés et causée par deux types d'herpèsvirus : l'herpèsvirus équin 1 (EHV1) et l'herpèsvirus équin 4 (EHV4). L'EHV 1 est plus important sur le plan médical et économique. Il peut causer des lésions respiratoires, des avortements $(1,12,13)$, des mortinatalités, des mortalités néonatales (6) et des lésions nerveuses (11). Quant à l'EHV4, il n'est en général à l'origine que de symptômes respiratoires et parfois d'avortements sporadiques.

L'infection naturelle, ainsi que la vaccination contre EHV1, induit une immunité partielle contre l'EHV4 $(4,5)$.

Après infection, les herpèsvirus équins peuvent persister à l'état latent dans l'organisme. Cet état de latence est à l'origine du maintien de l'infection dans un effectif sans nouvelle introduction de

1. Département de Pathologie Médicale et Chirurgicale des Equidés et Carnivores, Institut Agronomique et Vétérinaire Hassan II, Rabat, Maroc

Tél./fax : +2127771395

2. Département de Virologie, Société de Productions Biologiques et Pharmaceutiques Vétérinaires (BIOPHARMA), Rabat, Maroc

* Auteur pour la correspondance virus (3). Ceci, associé à la complexité de la pathogénie des infections herpétiques, rend difficile l'adoption d'une stratégie de prophylaxie médicale.

Le diagnostic sérologique se base sur les tests de séroneutralisation et de fixation du complément qui sont incapables de différencier entre les deux virus. Les tests ELISA qui ont l'avantage d'être spécifiques et rapides ont été développés récemment (9). Toutefois, le meilleur moyen pour différencier entre les infections herpétiques reste l'isolement et l'identification du virus en cause.

La présente étude se propose de faire le point sur l'importance des infections herpétiques dans la population équine marocaine. En outre, en la comparant avec des études réalisées antérieurement, elle a pour objectif de mettre en évidence les tendances évolutives de ces infections au Maroc.

\section{MATERIEL ET METHODES}

\section{Echantillonnage des sérums}

L'enquête a concerné 4775 sérums $(59,6$ p. 100 de chevaux, 18 p. 100 de mulets et 22,4 p. 100 d'ânes). Les sérums ont été prélevés entre 1990 et 1994 au cours d'enquêtes réalisées dans le 
cadre de l'épidémiosurveillance de la peste équine et au niveau des stations de monte afin de réaliser l'hémotypage des chevaux. Le programme d'hémotypage concernait les chevaux appartenant aux cinq haras nationaux, les juments des clubs équestres et celles qui ont été saillies au niveau des stations de monte, ainsi que leurs produits. Tous ces animaux seront conventionnellement désignés comme « chevaux des haras ».

Les sérums ont été prélevés dans plusieurs régions du territoire marocain et ont concerné des équidés de différents âges.

\section{Analyse sérologique}

\section{Souche virale}

La souche Kentucky de l'herpèsvirus équin 1 (EHV1) a été utilisée. Elle a été aimablement fournie aux auteurs par le Dr S. Zientara du Laboratoire central de recherches vétérinaires (CNEVA, MaisonsAlfort, France). Le virus a été produit sur lignée cellulaire RK13 en flacons Rollers. Le titre obtenu était de 6,2 $\log$ DECP50/ml. La dilution virale a été calculée de façon à avoir 100 DECP50 par cupule de sérum lors de la réaction de séroneutralisation.

\section{Culture cellulaire}

La lignée cellulaire RK13 a été utilisée à son $21^{\mathrm{c}}$ passage. La culture cellulaire a été entretenue dans un milieu de croissance à base de MEM additionné de sérum de veau foetal et d'antibiotiques. La suspension cellulaire pour la neutralisation a été obtenue par trypsination d'un tapis confluent et la concentration des cellules a été ajustée à 150000 cellules par millilitre.

\section{Test de séroneutralisation}

Pour détecter les anticorps spécifiques de l'herpèsvirus équin, le test de séroneutralisation en microméthode sur culture cellulaire a été utilisé. Les sérums étaient inactivés à $56^{\circ} \mathrm{C}$ pendant $30 \mathrm{~min}$ avant de procéder à un sondage à unc sculc dilution.

A un volume de $50 \mathrm{ml}$ contenant le sérum dilué au 1/3 ont été ajoutés $50 \mathrm{ml}$ (100 DECP50) de suspension virale. Le mélange a été incubé à $37^{\circ} \mathrm{C}$ pendant $60 \mathrm{~min}$ avant d'ajouter la suspension cellulaire. La lecture, détection de l'effet cytopathogène du virus EHV1 sur les cellules RK 13, a été réalisée cinq jours après infection. Le sérum était considéré comme positif s'il y avait neutralisation de cet effet.

\section{RESULTATS}

Sur les 4775 sérums analysés, 1857 , soit 38,89 p. 100 , se sont révélés positifs. Lors de cette étude, plusieurs facteurs de variation de la séroprévalence ont été considérés.

\section{L'espèce}

Les ânes avaient la séroprévalence la plus élevée (46,14 p. 100), suivis par les mulets $(41,96$ p. 100), puis les chevaux (35,35 p. 100) (figure 1). Il est à noter, cependant, qu'en 1990, les chevaux et les mulets avaient presque la même séroprévalence (tableau I). Ceci vient du fait que 25,9 p. 100 des sérums de chevaux prélevés cette année-là provenaient de la région d'El Kalaâ où un pourcentage de séropositivité très élevé a été constaté $(71,94$ p. 100), alors que seulement 10,6 p. 100 des sérums de mulets prélevés la même année provenaient de cette région. Par ailleurs, en 1992, les mulets étaient légèrement plus infectés que les ânes (tableau I). Mais 33,6 p. 100 des ânes étudiés cette année-là appartenaient à la zone Nord où le taux d'infection était relativement faible (24,75 p. 100).

\section{L'âge}

La séroprévalence augmentait avec l'âge jusqu'à la $3^{\mathrm{e}}$ année et restait sensiblement la même au-delà de cet âge (tableau II, figure 2).

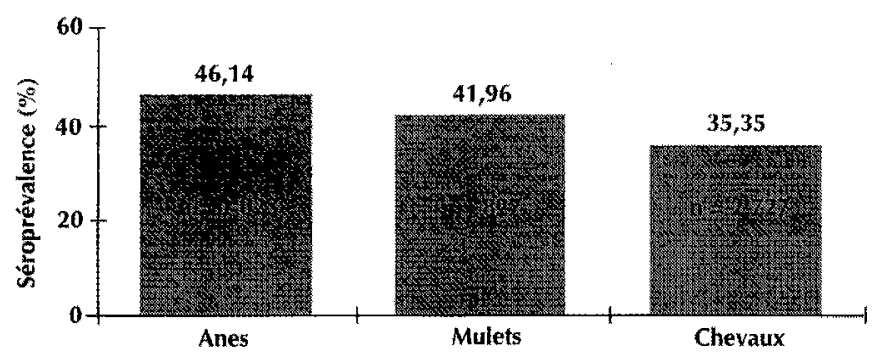

Figure 1 : séroprévalencc de la rhinopncumonic par cspècc.

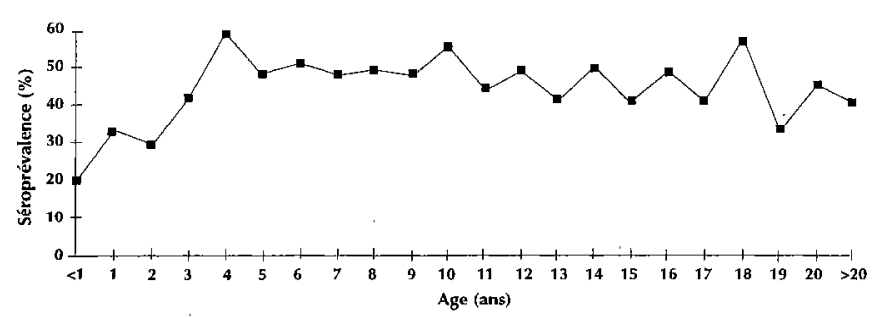

Figure 2 : séroprévalence de la rhinopneumonie en fonction de l'âge.

\section{Tableau I}

Séroprévalence de la rhinopneumonie par année et par espèce

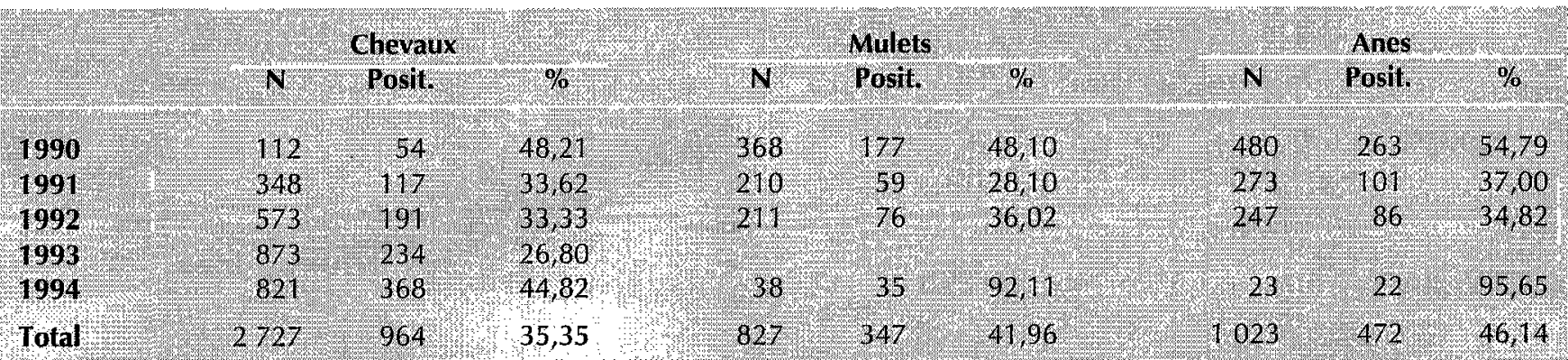




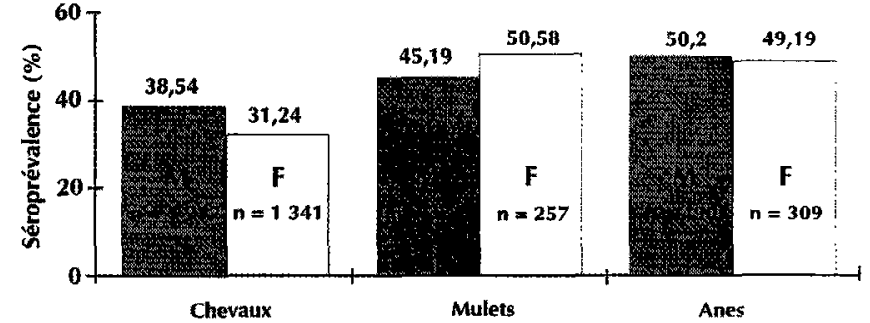

Figure 3 : séroprévalence de la rhinopneumonie par espèce et par sexe.

\section{Le sexe}

Chez les ânes et les mulets, les séroprévalences chez les deux sexes étaient sensiblement égales (figure 3), alors que chez les chevaux, les mâles étaient apparemment plus infectés que les femelles. Cependant, la ventilation des résultats par type d'élevage a révélé que la différence entre les séroprévalences chez les animaux des deux sexes était très minime (figure 4).

\section{Le type d'élevage}

Les chevaux des haras étaient moins infectés que les chevaux de trait (tableau III). De plus, la séroprévalence chez les chevaux des haras n'a subi que de légères variations durant les quatre années d'étude, alors que celle chez les chevaux de trait était irrégulière selon des années (tableau III).

\section{La région}

La séroprévalence la plus élevée a été relevée dans la région Sud $(56,25$ p. 100) (figure 5), bien que dans cette région la densité des équidés par $\mathrm{km}^{2}$ ait été la plus faible (tableau IV). Toutefois, le nombre d'échantillons prélevés dans cette région était faible (48 sérums). Dans les zones du Nord et de Tensift, les taux d'infection (respectivement 46,84 et 47,88 p. 100) étaient plus élevés que dans les zones Centre-Nord et orientale (respectivement 36,49 et 38,36 p. 100). La région centrale et la région Centre-Sud avaient les séroprévalences les plus basses (respectivement 28,46 et 30,09 p. 100), et ceci malgré le fait que la densité animale au niveau de la région centrale $\left(9,9\right.$ équidés $\left./ \mathrm{km}^{2}\right)$ ait été relativement importante (tableau IV).

Les ânes provenant de la zone de Tensift étaient largement plus infectés (71,62 p. 100) que ceux des autres régions (tableau IV). Par ailleurs, ces ânes avaient une densité respectivement 4 fois et 14 fois plus élevée que celle des mulets et des chevaux. D'autre part, les chevaux de la région Nord étaient largement plus infectés $(53,88$ p. 100$)$ que ceux des antres zones. Les prélèvements effectués dans la zone Centre-Sud appartenaient essenticllement ( 95 p. 100) aux chevaux de la zone d'action du haras de Meknès.

\section{L'année de prélèvements}

La séroprévalence paraissait avoir tendance à diminuer entre 1990 et 1993, puis a augmenté subitement en 1994 (figure 6). En fait, les prélèvements effectués en 1993 concernaient uniquement les chevaux des haras qui étaient moins infectés. Ces derniers ne figuraient pas parmi la population étudiée en 1990. Durant cette même année, les prélèvements efrectués che $\iota$ les ânes et les mulets (animaux plus prédisposés) étaient proportionnellement beaucoup plus nombreux que ceux effectués chez les chevaux.

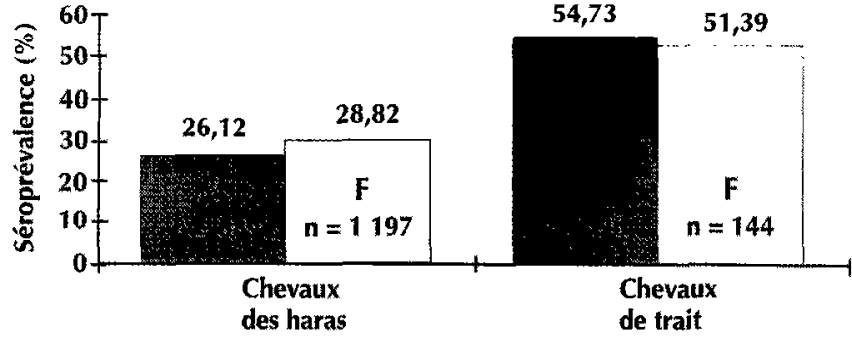

Figure 4 : séroprévalence de la rhinopneumonie par sexe et par type d'élevage.

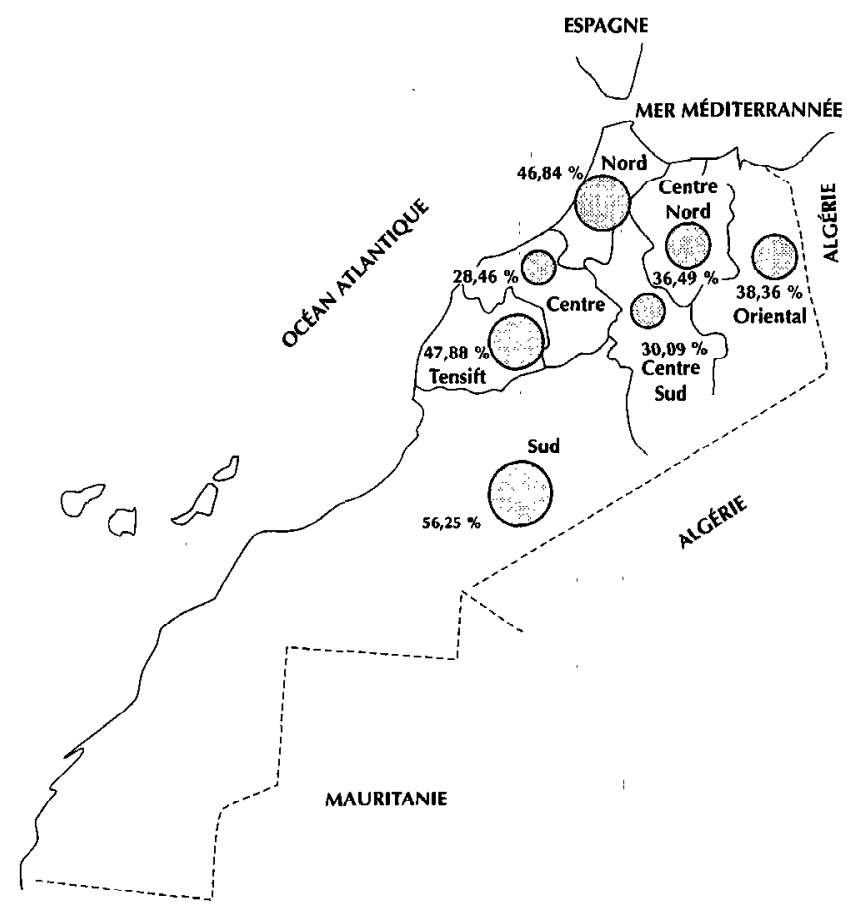

Figure 5 : séroprévalence de la rhinopneumonie par zone économique au Maroc.

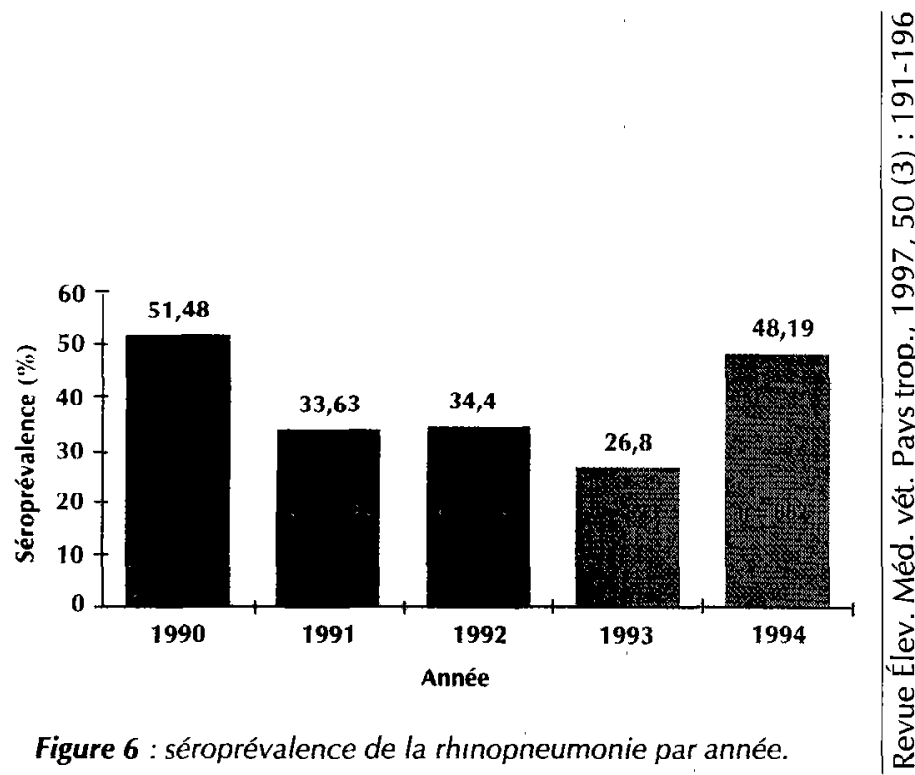




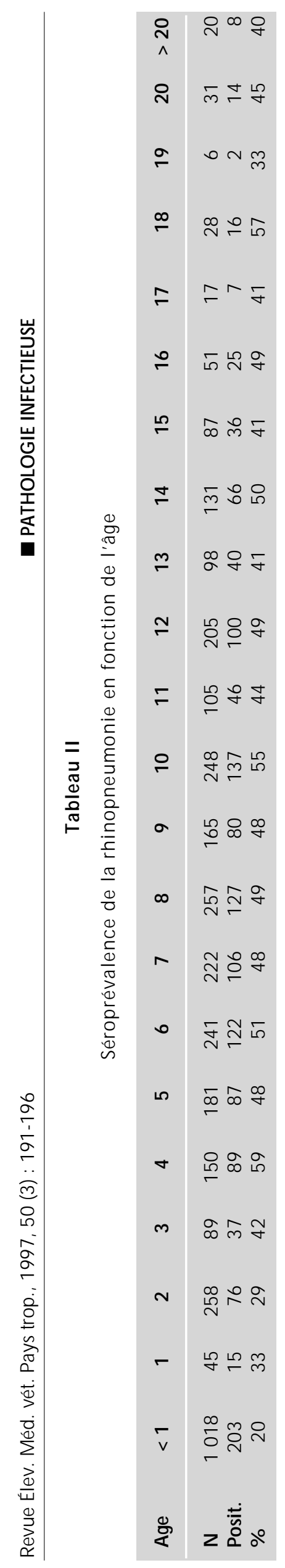

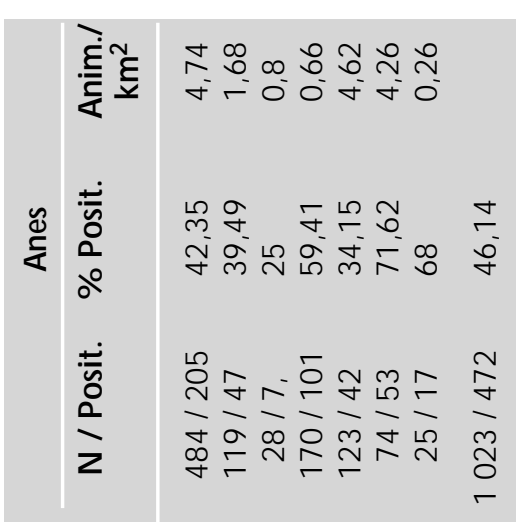

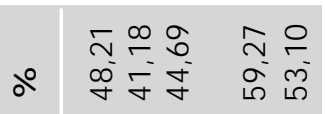

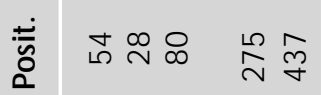

$\mathbf{z}$

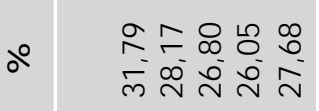

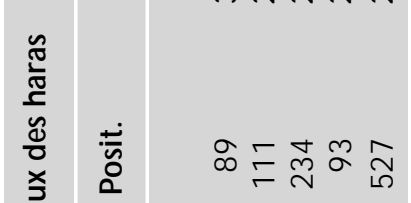

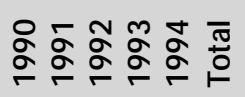

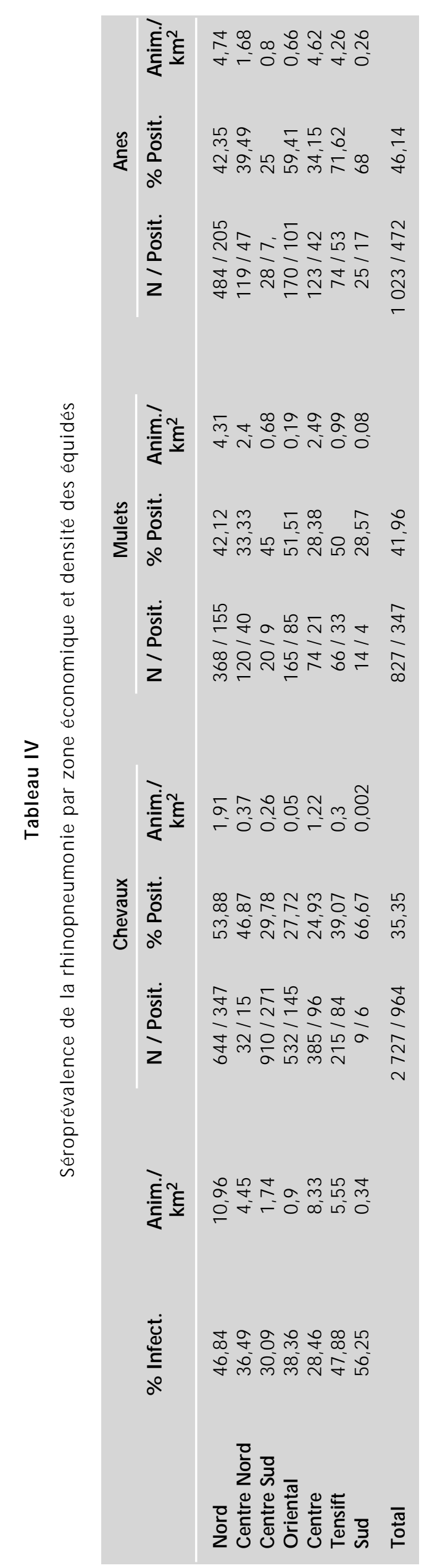




\section{DISCUSSION}

La détection des anticorps neutralisants signifie que les animaux ont été en contact avec le virus lors d'une infection naturelle ou par vaccination. La vaccination contre la rhinopneumonie équine n'est pas une pratique courante au Maroc et se limite à quelques chevaux de certains haras privés. Puisque ces animaux n'ont pas fait l'objet de cette étude, les résultats positifs obtenus étaient donc le reflet d'une infection naturelle. Lors de cette enquête, le test de séroneutralisation a été mis en œuvre et le virus utilisé était une souche d'EHV1 (souche Kentucky). Or, cette réaction ne permet pas de faire une distinction entre les deux sérotypes d'herpèsvirus équins EHV1 et EHV4. Dans ces conditions, les sérums positifs, au cours de cette étude, pouvaient être le reflet soit d'une infection par EHV1, soit par EHV4, soit par les deux à la fois (9).

Les anticorps neutralisants ont été détectés chez une large proportion des équidés étudiés (38,89 p. 100). Ce pourcentage est légèrement inférieur à ceux trouvés par Lahlou-Kassi (8) et Ourahma (10) qui étaient respectivement de 44,83 et 51,50 p. 100. Il est cependant supérieur à celui trouvé par Himer (7), soit 32,38 p. 100. Ces trois résultats ont été obtenus par le test de fixation du complément. Les pertes économiques dues aux avortements et aux mortalités néonatales au Maroc ne sont pas connues. Mais en se basant sur les résultats trouvés dans d'autres pays $(2,14)$, ces conséquences doivent être prises en compte sérieusement.

La séroprévalence a considérablement varié en fonction des différentes catégories d'animaux, et ainsi différents facteurs sont à considérer dans l'interprétation des résultats.

\section{L'espèce}

Les ânes avaient tendance à être plus prédisposés que les mulets tandis que les chevaux avaient le taux d'infection le plus bas. Le même résultat a été trouvé par Ourahma (10). Il est tentant d'expliquer ce fait par les différences de densité animale entre les trois espèces (le nombre d'ânes par km² était supérieur à celui des mulets et des chevaux). Or, les résultats trouvés en fonction de l'origine géographique ne soutiennent pas cette hypothèse, car les séroprévalences n'étaient pas en relation avec la densité des animaux dans les zones étudiées.

Le mode d'élevage des ânes et des mulets se caractérise par des rassemblements assez fréquents, notamment les jours de souk. Le contact direct est un facteur favorisant la transmission virale (3). En outre, le manque d'hygiène et la sous-alimentation dont souffraient les ânes et les mulets pouvaient contribuer à augmenter leur réceptivité.

\section{L'âge}

La probabilité pour que les équidés contractent le virus de la rhinopneumonie augmente avec l'âge. Ceci pourrait expliquer l'augmentation régulière de la séroprévalence jusqu'à l'âge de trois ans. Le fait que l'immunité faisant suite à la forme respiratoire qui touche les jeunes animaux soit de courte durée par rapport à celle produite par la forme abortive pourrait aussi expliquer cette augmentation. Au-delà de trois ans, il n'y a plus de prédisposition à l'infection liée à l'âge des animaux.

\section{Le sexe}

Le taux d'infection des mâles et des femelles était sensiblement le même chez les trois espèces. Par conséquent, le facteur sexe n'a pas d'influence sur la prédisposition à l'infection par les agents de la rhinopneumonie équine. Des résultats analogues ont été trouvés lors d'études précédentes menées au Maroc $(7,8,10)$.

\section{Le type d'élevage}

Le mode d'élevage des chevaux de trait se caractérise par une hygiène défectueuse, une alimentation insuffisante et une utilisation abusive, exception faite pour les chevaux de Fantasia. Par contre, la plupart des chevaux des haras bénéficient de conditions d'élevage favorables. Ces facteurs pourraient augmenter la réceptivité des chevaux de trait aux virus de la rhinopneumonie.

Par ailleurs, et contrairement aux chevaux des haras, les chevaux de trait vivent souvent à promiscuité d'autres équidés, notamment les ânes et les mulets qui sont plus réceptifs et par conséquent plus fréquemment infectés qu'eux.

\section{La région}

Etant donné le mode de transmission de l'EHV1 et de l'EHV4 qui se fait essentiellement par contact direct, on pourrait s'attendre à ce que les régions où la population équine était plus dense aient été les plus infectées. Or, la distribution des séroprévalences en fonction des régions n'était pas corrélée à la densité animale. Au niveau de la zone centrale où la densité des équidés était relativement importante par rapport aux autres zones du territoire marocain, des séroprévalences très faibles ont été relevées pour les trois espèces d'équidés. La séroprévalence la plus élevée a été relevée au niveau de la zone Sud. Cependant, le nombre de prélèvements effectués dans cette région était faible.

Cette enquête montre que les chevaux des haras étaient les animaux les moins infectés. Du fait que la majorité des prélèvements effectués au niveau de la zone Centre-Sud provenait de chevaux de l'aire d'action du haras de Meknès, cette région avait une séroprévalence plus faible. Les animaux qui se trouvaient au Nord et à Tensift avaient des séroprévalences supérieures à celles des équidés du Centre-Nord et de la zone orientale. Ceci est dû au fait qu'il $\mathrm{y}$ avait des régions où certaines espèces équines étaient curieusement plus infectées que d'autres. Il s'agissait des ânes se trouvant à Tensift et des chevaux se trouvant au Nord. A part ces deux foyers de la maladie, la prédisposition liée à l'origine géographique des animaux n'a pas été mise en évidence.

\section{L'année de prélèvements}

Au cours de l'année 1990, les prélèvements analysés ont été effectués sur les ânes, les mulets et les chevaux de trait. Par contre, en 1993, les prélèvements ont concerné uniquement les chevaux des haras. Ceci explique la différence entre les séroprévalences relevées pendant ces deux années. Pour les années 1991 et 1992, où l'effectif et la composition des échantillons étaient similaires, des valeurs presque égales ont été relevées. Par conséquent, les animaux étaient certainement régulièrement infectés entre 1990 et 1993.

L'année 1994 s'est caractérisée par une pluviométrie importante et un hiver froid, ce qui pourrait expliquer l'élévation importante de la séroprévalence au cours de l'année. Ces changements climatiques n'ont pas touché les chevaux des haras qui étaient élevés dans des locaux leur permettant d'être à l'abri des aléas climatiques. La valeur élevée qui a été observée en 1994 pourrait aussi s'expliquer par la prépondérance des sérums provenant des chevaux de la zone Nord, animaux ayant présenté une séroprévalence importante dans l'échantillon analysé.

\section{- CONCLUSION}

La rhinopneumonie est une maladie enzootique, largement répandue chez les équidés au Maroc, avec un taux de séropositivité de 38,9 p. 100. 
L'espèce et le mode d'élevage sont des facteurs qui jouent un rôle important dans la prédisposition à l'infection. En effet, les ânes (46,14 p. 100) sont plus prédisposés que les mulets $(41,96$ p. 100) et les chevaux (35,35 p. 100). Parmi ces derniers, les chevaux de trait sont plus touchés que les chevaux des haras.

Durant les cinq années de l'étude, il n'y a pas eu d'évolution importante de la maladie. Même si deux foyers ont été notés dans les zones Nord et Tensift, cette étude n'a pas révélé de différence entre les régions dans la prévalence de l'infection.

L'âge jouerait un rôle seulement chez les animaux de moins de trois ans.

\section{Remerciements}

Les auteurs remercient Biopharma pour les moyens matériels et humains engagés dans la réalisation de ce travail ; le Laboratoire des groupes sanguins, département de Pathologie médicale et chirurgicale des équidés et carnivores de l'Institut agronomique et vétérinaire Hassan II, de leur avoir fourni une partie des sérums analysés ; la Division des haras, Direction de l'élevage (MAMVA), pour avoir mis à leur disposition les statistiques de la population équine.

\section{BIBLIO GRAPHIE}

1. BRYANS J.-T., 1969. On immunity to disease caused by equine herpes virus 1. J. Am. vet. Med. Assoc., 155: 294-300.

2. CAMBELL T.-M., STUDDENT M.-J., 1983. Equine herpes virus type 1 (EHV1). Vet. Rec., 53: 135-143.

3. DELANNOY I., DOBOURGET Ph., FAYET G., 1995. Le point sur la rhinopneumonie. Prat. vet. équine, 27 : 31-47.

4. EDINGTON N., BRIDGES C.-G., 1990. O ne way protection between equid herpes virus 1 and 4 in vivo. Res. vet. Sci., 48: 223-239.

\section{Summary}

H midouch A., El Harrak M., Chakri A., O uragh L., Lotfi C., Bakkali-Kassimi L. Epidemiological study of rhinopneumonitis in Equidae in Morocco

During a serological survey of rhinopneumonitis in Moroccan equids over a five year period (1990 to 1994) 4775 samples (59.6\% horses, $18 \%$ mules and $22.4 \%$ donkeys) were collected in different regions of the country. The overall infection prevalence was $39 \%$. Donkeys (46\%) were more affected than mules (42\%) and horses (35\%). In addition, the seroprevalence increased with age in animals up to three years old in the three species to reach $42 \%$ and became stable in older animals. It was not clear whether the gender and the region they lived in predisposed them to the disease. However, maintenance conditions constituted a predisposing factor. After a stabilization period over the first four years, the incidence of the infection suddenly increased in 1994 because of the drought that occurred that year.

Key words: Horse - Ass - Mule - Equidae - Equine rhinopneumonitis virus - Immunological technique - Disease prevalence - Disease surveillance - Morocco.
5. ELLIS J.-A., BOGDAN J.-R., KANARA P.-S., HAINES D.-M., 1995. Cellular and antibody responses to equine herpes viruses 1 and 4 following vaccination of horses with modified-live and inactivated viruses. JAVM A, 206: 823-832.

6. HARTLEY W.-J., DIXON R.-J., 1979. An outbreak of foal perinatal mortality due to equine herpesvirus type 1: pathological observations. Equine vet. J., 11: 215-218.

7. HIMER D., 1975. Incidence au Maroc de l'anémie infectieuse, de la rhinopneumonie et de l'artérite virale des équidés : enquête épidémiologique - prophylaxie. Thèse doct. vét., Ecole nationale vétérinaire, Maisons-Alfort, France.

8. LAHLOU-KASSI S., 1977. Enquête épidémiologique sur I'anémie infectieuse, la rhinopneumonie et l'artérite à virus des équidés au Maroc. Thèse doct. vét., Institut agronomique et vétérinaire Hassan II, Rabat, Maroc.

9. MUMFORD J.-A., 1995. Virus infections regarding the respiratory tract. In: Proc. 4th Geneva congress of equine medicine and surgery, Geneva, Switzerland, December 10-12, 1995.

10. OURAHMA E., 1984. Contribution à l'étude épidémiologique des maladies virales respiratoires des équidés au Maroc. Thèse doct. vét., Institut agronomique et vétérinaire Hassan II, Rabat, Maroc.

11. PLATT H., SINGH H., WHITHWELL K.-E., 1980. Pathological observations on an outbreak of paralysis in brood mares. Equine vet. J., 12: 118-126.

12. POWELL D.-G., BURROWS R., SPO ONER P.-R., GOODRIDGE D., THOMSON G.-R., MUMFORD J., 1978. A study of infectious respiratory diseases among horses in Great Britain, 1971-1976. In: Bryans J.-T., G erber H. eds., Int. conf. equine infectious diseases, Lyon, 1976, vol. 4. Princeton, NJ, USA, Veterinary Publications, p. 451-459.

13. STUDDERT M.-J., 1974. Comparative aspects of equine herpesviruses. Cornell Vet., 64: 94-122.

14. STUDDERT M.-J., FITZ PATRICK D.-R., HORNER G.-W., WESTBU RY H.-A., GLEESO N L.-J., 1984. Molecular epidemiology and pathogenesis of some equine herpes virus type 1 (equine abortion virus) and type 4 (equine rhinopneumonitis virus) isolates. Austr. vet. J., 61: 345-348.

Reçu le 2.2.97, accepté le 8.10.97

\section{Resumen}

H midouch A., El Harrak M., Chakri A., O uragh L., Lotfi C., Bakkali-Kassimi L. Estudio epidemiológico de la rinoneumonía en los equinos de Marruecos

Se llevó a cabo una encuesta serológica sobre la rinoneumonía equina en Marruecos, con 4775 muestras $(59,6 \%$ caballos, $18 \%$ mulas y $22,4 \%$ asnos), durante cinco años (de 1990 a 1994) en las diferentes regiones del país. La seroprevalencia global de la infección fue de 39\%. Los asnos $(46 \%)$ fueron más afectados que las mulas $(42 \%)$ y los caballos (35\%). Por otro lado, en las tres especies la seroprevalencia aumentó con la edad, hasta los tres años, alcanzando un valor de $42 \%$, estabilizándose en los animales de mayor edad. La predisposición según el sexo de los animales y de la región geográfica en donde estos vivían no fue evidente. Las condiciones de mantenimiento, por el contrario, constituyeron un factor predisponente para la infección. Después de una estabilización durante los cuatro primeros años, la incidencia de la infección aumentó súbitamente en 1994, debido a la sequía que se vivió ese año.

Palabras clave: Caballo - Asno - Mula - Equidae - Virus de la rinoneumonitis equina - Técnica immunologíca - Prevalencia de una enfermedad - Vigilancia de enfermedades Marruecos. 